\title{
Thin Film Deposition and Modification of Fullerenes by Laser Radiation
}

\author{
Prof. Dr. rer. nat. Asta Richter and Dr. rer. nat. Klaus-Werner Brzezinka
}

\section{Zusammenfassung}

Mit einem gepulsten $\mathrm{CO}_{2}$-Laser wird Laserablation von festen Fullerene-Pellets und texturierten Graphittargets zur Abscheidung von dünnen Fullerite- und diamantartigen Kohlenstoffilmen (DLC) getestet. Ablation von Fullerene-Material ist nicht zur Bildung von Fullerite-Filmen geeignet, sondern es entstehen amorphe pregraphitische Kohlenstoffstrukturen. Schäden, die durch die Einwirkung des Lasers auf Fullerene verursacht werden, wurden mittels Ramanspektroskopie untersucht. Bei geringen Laserleistungsdichten werden Strukturänderungen mit wachsender Einwirkungszeit des Lasers beobachtet. Diese thermischen Effekte können das Fullerene-Material nicht vollständig umwandeln, ohne daß die Laserleistungsdichte erhöht wird. Thermische Verdampfung ist eine geeignete Methode, um FulleriteSchichten guter Qualität abzuscheiden; so konnten epitaktische Filme auf Glimmer aufwachsen. $\mathrm{C}_{60}$ /DLC-Sandwich-Strukturen werden durch Laserabscheidung hergestellt und zeigen scharfe Grenzflächen, wie aus der definierten Überlagerung der charakteristischen Ramanspektren des Fullerite- und DLC-Films abgeleitet werden kann.

\begin{abstract}
Laser ablation from solid fullerene pellet and textured graphite targets has been probed for deposition of thin fullerite and diamond-like carbon (DLC) films by means of a pulsed $\mathrm{CO}_{2}$ laser: Ablation of fullerene material does not yield fullerite films, but results in amorphous pregraphitic carbon structures. At very low laser flux structural transformations are observed with increasing exposure time. These thermal effects cannot completely decompose the fullerenes without increase of the laser power density. Thermal evaporation is a suitable method to deposite fullerite films of high quality, thus epitaxial fullerite films are grown on mica. Sandwich $C_{60} / D L C$ structures are produced by laser deposition which show a sharp interface as can be deduced from the superposition of the characteristic Raman spectra of the fullerite and the DLC.
\end{abstract}

\section{Introduction}

Fullerenes and various carbonaceous materials have attracted much attention because of their numerous potential applications in different areas of physics and chemistry. Surface coatings on carbon base are promising since they combine under certain conditions interesting tribological features as high hardness, high corrosive resistance, and solid lubrication. Moreover, optical and electronic properties of carbonaceous materials open a field in novel applications. Thus, a large number of carbon based coatings has been developed, ranging from graphite via polymeric and amorphous diamond-like carbon (DLC) to diamond and fullerite films [e. g. 1-6]. Fullerenes as a new class of carbon are interesting either because of their inherent properties, but also because of a certain possibility to transform them into other carbonaceous forms. Formation of sandwich and multilayer structures including fullerite films of a few monolayers or thick films gives rise to novel applications in the field of thin film technology. The weakly bonded $\mathrm{C}_{60}$ molecule is expected to generate a new type of interface. Moreover, fullerite could reduce the strain in the interface in a material combination containing a highly stressed film. Combination of different forms of carbon are interesting since they allow the generation of a new mixing of outstanding properties of the single carbon forms. The sheroidic $\mathrm{C}_{60}$ molecules open another dimension of structure formation in carbonaceous materials.

Besides others laser ablation for thin film deposition is a promising new method in the wide field of film growth of tailor made recent materials and material combinations $[7,8]$. Film growth during the pulsed laser deposition process is facilitated by condensation of a plasma generated by the interaction of a pulsed laser beam with a solid target. DLC films [9] and high $\mathrm{T}_{\mathrm{C}}$ superconducting YBCO films [10] are excellent examples for thin film growth of high quality with this method. The main advantage of this technique is the ejection of various excited and ionic species along with the neutral particles within the laser plasma. Because of the energy content of the laser plasma temperatures and pressures are generated which can cause structural transformations as in the case of carbonaceous materials. Moreover, particles arriving with the plasma on the substrate surface intermix with the substrate atoms, thus the adherence of the films is improved. Gas supply into the deposition chamber allows reactive processes which are important for deposition of oxides or nitrides.

Raman scattering is a unique tool to characterize different kinds of ordered and disordered carbon $[9,11]$ and used as a "fingerprint" method. Raman scattering depends on the size of graphite crystallites and on the degree of $\mathrm{sp}^{2}$ and $\mathrm{sp}^{3}$ bonding. Fullerite has a rich Raman spectrum relative to other materials because of the 
molecular nature of $\mathrm{C}_{60}$ and the high symmetry of the molecule.

In this contribution we present results on the laser deposition process of fullerite films and the modification of fullerenes by the interaction with laser radiation. $\mathrm{C}_{60}$ /DLC sandwich structures are deposited and investigated by Raman spectroscopy.

\section{Laser Ablation Process}

The discovery of $\mathrm{C}_{60}$ clusters is highly correlated with the laser ablation process. Graphite can be laser vaporized into a carrier gas stream. The obtained carbon cluster size distribution was observed and analysed by Smalley and co-workers [12] and appeared to consist of two distributions: a low-mass region in which both odd and even clusters are represented ending at about 20 atoms and a high-mass region beginning at about 40 atoms consisting of only even clusters. The clusters in the second distribution are closed-cage fullerenes with a high intensity of the $\mathrm{C}_{60}$ clusters. However, the amount of $\mathrm{C}_{60}$ to produce macroscopic samples by laser vaporization of graphite is too small.

We obtained raw fullerene powder by the standard method proposed by Krätschmer et all. [13], i. e. the evaporation of graphite rods in a 1.5 to $2.0 \times 10^{4} \mathrm{~Pa}$ helium atmosphere using an electric arc welder [6]. The outgased blackish $\mathrm{C}_{60}$ powder was used as starting material for thin film deposition.

The principle set-up for the laser ablation process is shown in fig. $1 . \mathrm{A} \mathrm{CO}_{2}$ laser of $10.6 \mu \mathrm{m}$ wave length and a variable pulse length is used. The shortest pulse length of the laser being 100 ns resluts in a maximum energy density of $5 \mathrm{~J}$. The laser beam is focussed to a spot of about 1 to $2 \mathrm{~mm}^{2}$ giving a maximum laser power density of $5 \times 10^{9} \mathrm{~W} / \mathrm{cm}^{2}$. The basic pressure in the chamber was of about $10^{-4} \mathrm{~Pa}$. Two solid rotating targets are positioned in the chamber in order to change between two materials during the ablation process. One target was textured pure graphite, the other one was made from the raw fullerene powder as a pellet pressed at room temperature. The $C_{60}$ pellet was investigated by $x$-ray diffraction and Raman spectroscopy. The x-ray spectrum

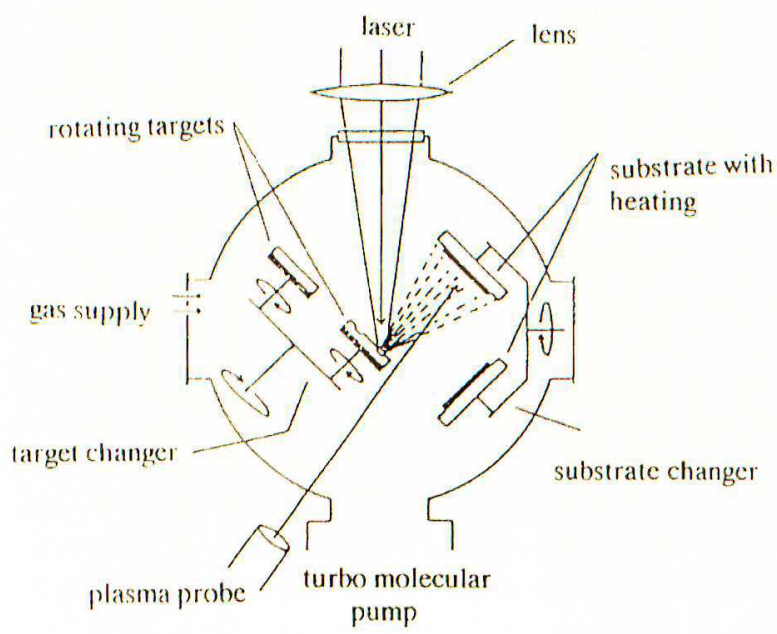

Fig. 1: Principle set-up of the laser deposition facility of the fullerene pellet is dominated by the $111,220,311$, and 222 Bragg peaks, which are assigned to a fcc lattice with nearest neighbour distance between two cage centers of $1.003 \mathrm{~nm}$ and a lattice constant of $1,419 \mathrm{~nm}$. Additional reflections up to an angle of $2 \Theta=85^{\circ}$ confirm the fcc lattice.

Raman spectra were measured over a large frequency range of 100 to $3200 \mathrm{~cm}^{-1}$ at room temperature with the $514 \mathrm{~nm}$ radiation of an $\mathrm{Ar}^{+}$laser for excitation [6]. All ten characteristic Raman active modes for the $C_{60}$ molecule are observed as shown in fig. 2. Additional features at 528,974 , and $1309 \mathrm{~cm}^{-1}$ are attributed to the silent modes $\mathrm{F}_{1 \mathrm{u}}(1), \mathrm{F}_{1 \mathrm{~g}}(2)$, and $\mathrm{G}_{\mathrm{u}}(5)$, respectively. Crystal fields in the small fullerite crystals and/or isotopic enrichment of ${ }^{13} \mathrm{C}$ effectively lower the symmetry of the $\mathrm{C}_{60}$ molecules, thus the forbidden Raman modes become active in the solid state [6].

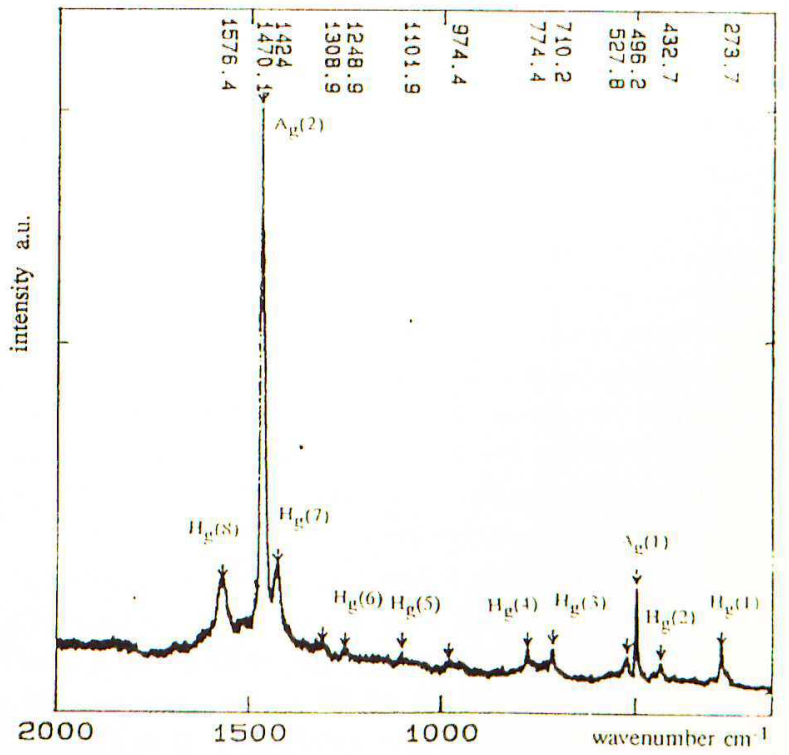

Fig. 2: Typical first-order Raman spectrum of a pure $C_{60}$ pellet

Ablation of the $C_{60}$ pellets under the described conditions does not result in fullerite films, but in amorphous carbon films with a pregraphitic structure. The film surface is inhomogenous without a good adherence. Craters of plasma interaction with melting traces and sprayed particles are visible (fig. 3a). The small areas of layers formed contain internal stresses, thus wrinkles are caused with debonded areas of the film (fig. 3b). Therefore, laser ablation is not a suitable method
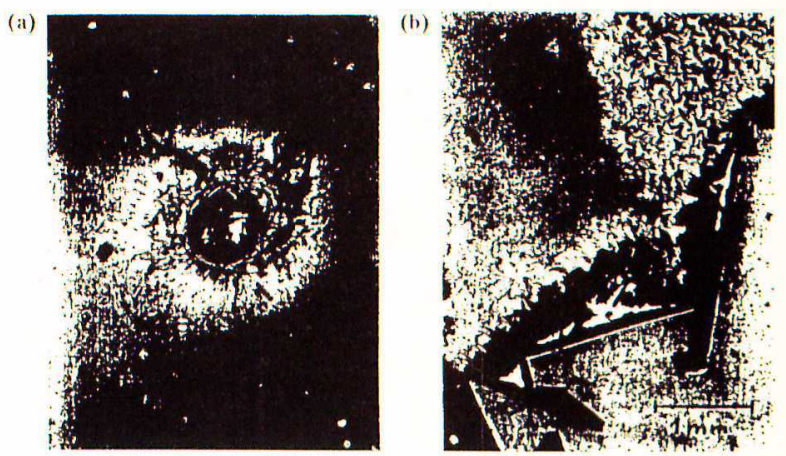

Fig. 3: Light microscopy of the film surface deposited by a laser ablation from a fullerene pellet: (a) crater of plasma interaction, (b) debonded region with wrinkles 
to grow fullerite films. The laser power density should be small with long laser pulses, that means fullerite films can be grown by thermal evaporation.

\section{Laser Damage Effects on Fullerite}

Damage effects of laser radiation on $\mathrm{C}_{60}$ material are carefully studied by systematic investigations with an $\mathrm{Ar}^{+}$laser. Exposure of fullerite to the laser beam with a very small power of 25 to $500 \mu \mathrm{W}$ on the sample surface at a spot of about $10 \mu \mathrm{m}^{2}\left(\Phi=25\right.$ to $\left.500 \mathrm{~kW} / \mathrm{cm}^{2}\right)$ results in time dependent structural changes as shown in fig. 4 . The frequency region around $1500 \mathrm{~cm}^{-1}$ in the Raman spectra mainly reflects fullerite decomposition. With an exposure time of the laser beam at the same surface spot less than 20 s the typical Raman spectrum with the dominating $A_{g}(2)$ line and the $H_{g}(7)$ and $H_{g}(8)$ lines at 1468,1421 , and 1572 is obtained. Athough the laser interaction with the fullerite was performed in air at room temperature no photopolymerization of the $C_{60}$ was observed. With increasing exposure time of the laser beam the $A_{g}(2)$ line is not shifted in frequency, but has decreased in intensity. The two $\mathrm{H}_{\mathrm{g}}$ modes are broadened and side bands grow at frequencies of about 1600 and $1400 \mathrm{~cm}^{-1}$. These side bands strongly increase with increasing exposure time of the laser radiation, so the $\mathrm{H}_{\mathrm{g}}$ modes cannot be detected within the rather broad and strong wings of the Raman features with a maximum at 1602 and $1405 \mathrm{~cm}^{-1}$ after $600 \mathrm{~s}$. We attribute these broad bands to the $\mathrm{G}$ and $\mathrm{D}$-line known from disordered carbons [9]. Thus disordered pregraphitic carbon is formed during the laser interaction. This means, the $\mathrm{C}_{60}$ molecules are deformed and transformed into small almost flat aromatic pregraphitic layers. However, this fragmentation process is not complete as the strong $A_{g}(2)$ feature for $C_{60}$ is still present. From the time dependent decomposition we assume that the material changes are caused by thermal processes. With increase of the exposure time without changes of the laser flux the Raman spectrum obtained for $600 \mathrm{~s}$ in fig. 4 does not change drastically. An increase of the laser power density results in a complete decomposition of the fullerene material. A tentative threshold value for laser damage

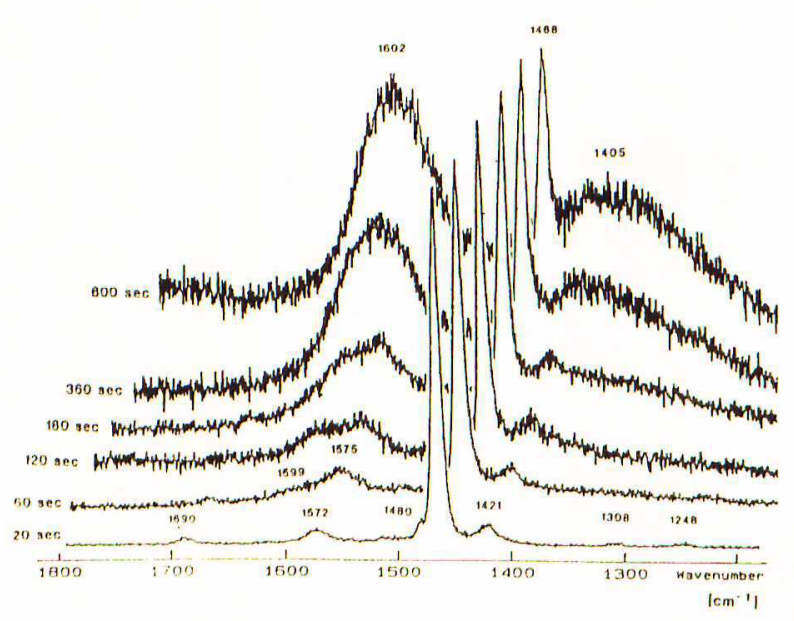

Fig. 4: Raman spectra of fullerite obtained with $500 \mathrm{~mW}$ laser power on the sample surface at different exposure times under the described experimental conditions is obtained with flux of $10^{7} \mathrm{~W} / \mathrm{cm}^{2}$. The damage process was not reversible in such a way that the fullerene features again appear if the laser flux is reduced.

\section{Thermal Evaporation of Fullerenes}

Thermal evaporation by laser means that a balance between the absorbed laser radiation which is transformed into heat and heat conduction from the laser spot into the sample is expected. With a sufficient, but low laser flux the $C_{60}$ molecules evaporate and attain the substrate surface with thermal energy. However, only a thin surface layer of the rotating pellet evaporates with laser radiation contrary to conventional evaporation technique. With power densities of about $10^{7} \mathrm{~W} / \mathrm{cm}^{2}$ fullerite films could be obtained. The polycrystalline films contain particles and droplets caused by the soft $\mathrm{C}_{60}$ target material during laser processing.

Thermal evaporation for growth of fullerite films is known to be a standard method [2-6]. Beside laser evaporation we deposited fullerite films on different substrates under UHV conditions at $10^{-5}$ to $10^{-7}$ Pa with an evaporation temperature of $450^{\circ} \mathrm{C}$ and substrate temperatures at 150 to $200^{\circ} \mathrm{C}$. The deposition rate was with $0.01 \mathrm{~nm} / \mathrm{s}$ low. The chamber pressure remained constant during the deposition process indicating that no volatile solvents were present in the raw fullerene powder. Polycrystalline fullerite films are detected on silicon whereas epitaxial films are grown on mica due to an inplane periodicity of the hexagonal sheets of $\mathrm{SiO}_{4}$ tetrahedra of $0.25 \mathrm{~nm}$ in mica which can be superimposed with the $\mathrm{C}_{60}$ molecules with a diameter of 1.04 $\mathrm{nm}|2|$. Thus, only the $\{\mathrm{III}\}$ family of Bragg reflexes is observed which shows that the $\{I I I\}$ fullerite planes are parallel to the substrate surface.

\section{5. $C_{60} /$ DLC Sandwich Structures}

Combination of fullerite and DLC films with a sharp interface was realized by laser deposition from a $C_{60}$ target followed by laser ablation from a graphite target. $\mathrm{Si}(111)$ substrates with the natural $\mathrm{SiO}_{2}$ layer are first covered with fullerite films of thickness 180 to $500 \mathrm{~nm}$ by thermal evaporation and then DLC coatings up to a thickness of $450 \mathrm{~nm}$ are deposited by laser ablation from a graphite target. $\mathrm{C}_{60}$ molecules are not destroyed by the DLC deposition process as can be seen from the clear superposition of the characteristic Raman spectra for $C_{60}$ and DLC films in fig. 5.

Very thin DLC coatings on the fullerite do not influence the typical rich Raman peak structure of the $C_{60}$ molecular solid. With increasing DLC film thickness the peak intensities caused by the fullerite are significantly diminished. Moreover, the $\mathrm{H}_{\mathrm{g}}(8)$ line is down shifted in frequency. For DLC layers thicker than $100 \mathrm{~nm}$ the broad characteristic Raman spectrum of DLC is superimposed with the dominant $A_{g}(2)$ line of $C_{60}$. The maximum of the broad asymmetric Raman band of DLC is at $1558 \mathrm{~cm}^{-1}$ and does not change with further increase in film 
thickness. The Raman spectrum of the $\mathrm{C}_{60}$ /DLC sandwich structure is completely different from the structural changes caused by laser damage in the fullerenes (compare figs. 4 and 5). The fullerite structure of laser damaged $\mathrm{C}_{60}$ materials changed into amorphous partly pregraphitic carbon and leavings of $\mathrm{C}_{60}$ fragments or molecules whereas the sandwich structures superimpose both structural properties characteristic for $\mathrm{C}_{60}$ and DLC.

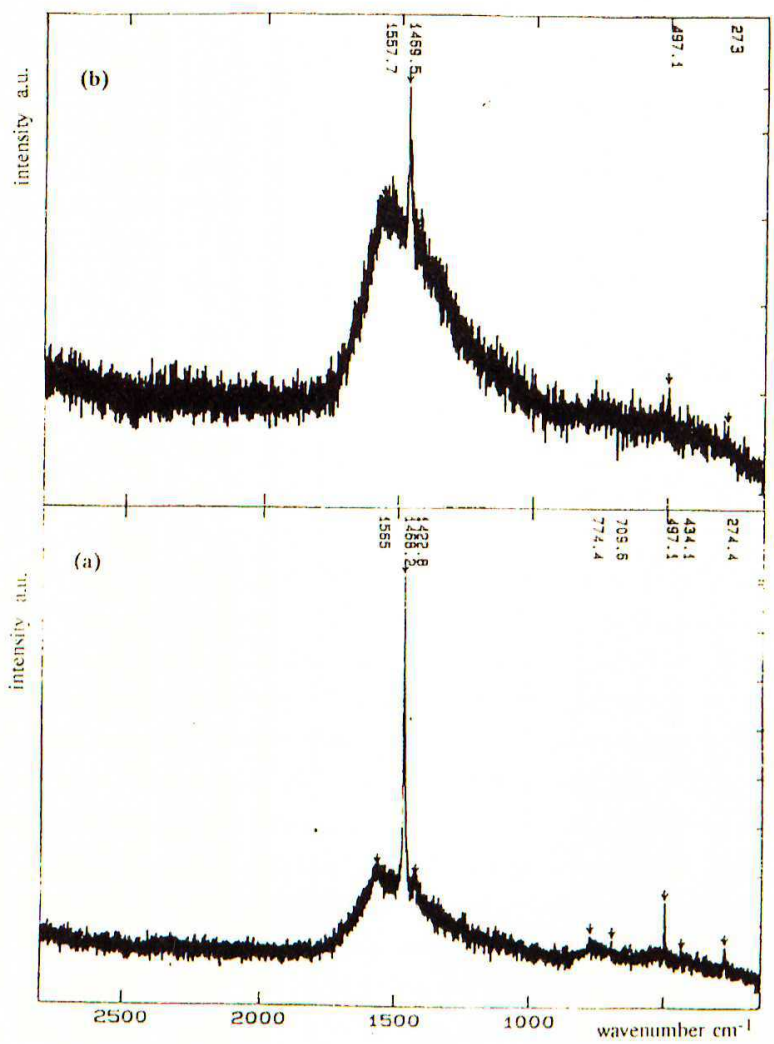

Fig. 5: Typical Raman spectra of C60/DLC sandwich structures on silicon with a thickness of the DLC coating of $70 \mathrm{~nm}$ (a) and $180 \mathrm{~nm}$ (b)

\section{References}

|11 R.D. Davis (ed.), Diamond Films and Coatings, Noyes, Park Ridge, NJ (1993).

[2] W. Krakow, N.M. Rivera, R.A. Roy, R.S. Ruoff, and J.J. Cuomo, J. Mater. Res. 7 (1992) 784.

|3| G. Gensterblum, L.-M. Yu, J.-J. Pireaux, P.A. Thiry, R. Caudano, J.-M. Themlin, S. Bouzidi, F. Coletti, and J.-M. Debever, Appl. Pliys. A 56 (1993) 175.

|4| H.-G. Busmann, R. Hiss, H. Gaber, and I.V. Hertel, Surface Science 289 (1993) 381.

[5] W.B. Zhao, X.D. Zhang, Z.Y. Ye, J.L. Zhang, C.Y. Li, D.L. Yin, Z.N. Gu, X.H. Zhou, and Z.X. Jin, Thin Solid Films 240 (1994) 14.

(6) A. Richter, K.-W. Brzezinka, P. Reich, J. Erxmeyer, B. Mertesacker, H.-J. Scheibe, and D. Drescher, submitted Phys. Rev. B.

[7] A. Richter and W. Pompe, J. Non-Crystalline Solids 97\&98 (1987) 1443.

|8| H.-J. Scheibe and B. Schultrich, Thin Solid Films 246 (1994) 92.

[9] A. Richter, H.J. Scheibe, W. Pompe, K.-W. Brzezinka, and 1. Mühling, J. Non-Crystalline Solids 88 (1986) 131.

A. Richter and M. Klose, Optics \& Laser Technology 24 (1992) 215.

[10] E.V. Pechen, S.I. Krasnosvobodtsev, G. Kessler; A. Richter, M. Panzner, O. Großmann, and A. Teresiak, phys. stat. sol. (b) 131 (1992) 179.

(11] P. Reich, K. Witke, and K.-W. Brzezinka, Fresenius J. Anal. Chem. 349 (1994) 76

|12 H.W. Kroto, J.R. Heath, S.C. O 'Brien, R.F. Curl, and R.E. Smalley, Nature 318 (1985) 162.

|13| W. Krätschmer, L.D. Lamb, K. Fostiropoulos, and D.R. Huffman, Nature 347 (1990) 354.

\section{Verfasser}

Prof. Dr. rer. nat. Asta Richter

Technische Fachhochschule Wildau

Fachbereich Physikalische Technik (Department of Physics and Technology)

Tel. +49 (0) 33 75/507-184

Dr. rer: nat. Klaus-Werner Brzezinka

Bundesanstalt für Materialforschung und -prifung, Unter den Eichen 87, D-12205 Berlin, Germany 